\title{
Writing with atoms: Oxygen adatoms on the
}

\section{$\mathrm{MoO}_{2} / \mathrm{Mo}(110)$ surface}

Sergey A. Krasnikov, ${ }^{, \dagger}$ Olaf Lübben, ${ }^{\dagger}$ Barry E. Murphy, ${ }^{\dagger}$ Sergey I. Bozhko, ${ }^{\dagger, t}$ Alexander N. Chaika, ${ }^{,+t}$ Natalia N. Sergeeva, ${ }^{\S}$ Brendan Bulfin, ${ }^{\dagger}$ and Igor V. Shvets ${ }^{\dagger}$

$\dagger$ Centre for Research on Adaptive Nanostructures and Nanodevices, School of Physics, Trinity College Dublin, Dublin 2, Ireland, *Institute of Solid State Physics, Russian Academy of Sciences, Chernogolovka 142432, Russian Federation, and ${ }^{\S}$ School of Chemistry, University of Leeds, Leeds LS2 9JT, UK.

AUTHOR EMAIL ADDRESS Sergey.Krasnikov@dcu.ie

RECEIVED DATE (to be automatically inserted after your manuscript is accepted if required according to the journal that you are submitting your paper to)

*To whom correspondence should be addressed. E-mail: Sergey.Krasnikov@dcu.ie

ABSTRACT

Writing at the nanoscale using the desorption of oxygen adatoms from the oxygen-rich $\mathrm{MoO}_{2+\mathrm{x}} / \mathrm{Mo}(110)$ surface is demonstrated by scanning tunneling microscopy (STM). High-temperature oxidation of the $\mathrm{Mo}(110)$ surface results in a strained, bulk-like $\mathrm{MoO}_{2}(010)$ ultra-thin film with an OMo-O tri-layer structure. Due to the lattice mismatch between the $\mathrm{Mo}(110)$ and the $\mathrm{MoO}_{2}(010)$, the latter consists of well-ordered molybdenum oxide nanorows separated by $2.5 \mathrm{~nm}$. The $\mathrm{MoO}_{2}(010) / \mathrm{Mo}(110)$ structure is confirmed by STM data and density functional theory calculations. Further oxidation results in the oxygen-rich $\mathrm{MoO}_{2+\times} / \mathrm{Mo}(110)$ surface, which exhibits perfectly aligned 
double rows of oxygen adatoms, imaged by STM as bright protrusions. These adatoms can be removed from the surface by scanning (or pulsing) at positive sample biases greater than $1.5 \mathrm{~V}$. Tip movement along the surface can be used for controlled lithography (or writing) at the nanoscale, with a minimum feature size of just $3 \mathrm{~nm}$. By moving the STM tip in a predetermined fashion, information can be written, read and erased by applying specific biases between the surface and the tip.

KEYWORDS: STM, inelastic tunneling, atom manipulation, molybdenum oxide

Johannes Gutenberg's system of movable type brought the written word to the masses in the 15 th century and changed the world. It catalyzed the Renaissance and the scientific revolution, and has led to many aspects of the modern world that are now taken for granted. Lithography techniques have undoubtedly come a long way since Gutenberg's time, but their importance to society is arguably no less monumental. Modern techniques, such as electron beam, ion beam and photo-lithography can produce feature sizes down to hundreds or tens of nanometers. This allows more complex and sophisticated integrated circuit architectures than ever before, however even these techniques have fundamental limits to their resolution. It is for this reason that the direct manipulation of atomic structures will likely be the next step in the evolution of lithography.

Soon after the invention of the scanning tunneling microscope (STM) in $1982,{ }^{1}$ researchers began using the unprecedented precision of the STM tip to deform atomically-flat samples. ${ }^{2,3}$ These first attempts at nanoscale lithography were quite crude, involving mechanical and thermal deformation of the substrate. However, they have inspired many more sophisticated approaches to this problem. The ability to drag individual adsorbate atoms by STM tips has been exploited in order to position atoms with subnanometer lateral precision, ${ }^{4-8}$ however in general the speed of the patterning was too slow and the fabricated atomic structures could survive only at liquid helium temperatures. Scanning probe 
lithography on the nanoscale can be performed in one of three modes: modification of the substrate, for example by resist exposure or oxidation; ${ }^{9-12}$ material deposition; ${ }^{13-17}$ or material removal or etching. ${ }^{18-20}$

The oxidation of metal surfaces can produce a variety of ordered oxide nanostructures and twodimensional surface oxides, ${ }^{21,22}$ which are of great significance for many applications including heterogeneous catalysis. ${ }^{23,24}$ In this paper a novel lithography system is described, in which an STM tip is used to remove single oxygen adatoms from the oxidised molybdenum surface, which exhibits wellordered oxide nanorows. The oxygen desorption is induced by electrons tunneling from the STM tip to the sample. The process can be initiated at positive sample bias voltages above $1.5 \mathrm{~V}$ without contact between the tip and the surface, substantially decreasing the probability of modifying the tip structure and therefore allowing reproducible atomic-scale patterning. Due to the high stability of the oxide system, the nanoscale writing reported herein does not require extremely low temperatures. Furthermore, the lateral size of the patterns on the oxidised $\operatorname{Mo}(110)$ surface can be controlled by the bias voltage applied during the STM lithography. The minimum feature size of this technique is $3 \mathrm{~nm}$, which is a fundamental constraint due to the periodicity of the molybdenum oxide nanorows on the $\operatorname{Mo}(110)$ surface. Such fine resolution is an order of magnitude improvement over even the most cutting-edge electron beam techniques, and represents an interesting avenue for the development of new procedures for future technologies.

The STM experiments were performed at liquid nitrogen temperature $(78 \mathrm{~K})$, using a commercial instrument from Createc, in an ultra-high-vacuum (UHV) system consisting of an analysis chamber (with a base pressure of $\left.2 \times 10^{-11} \mathrm{mbar}\right)$ and a preparation chamber $\left(5 \times 10^{-11} \mathrm{mbar}\right)$. An electrochemicallyetched [100]-oriented single crystalline W tip ${ }^{25,26}$ was used to record STM images in constant current mode. The voltage $\mathrm{V}_{\mathrm{b}}$ corresponds to the sample bias with respect to the tip. No drift corrections have been applied to any of the STM images presented in this paper.

A Mo(110) single crystal (Surface Preparation Laboratory) was used as the substrate. An atomically-clean $\mathrm{Mo}(110)$ surface was prepared by in situ annealing at $1500 \mathrm{~K}$ in an oxygen atmosphere 
of $1 \times 10^{-7} \mathrm{mbar}$, followed by a series of high temperature flashes at $2200 \mathrm{~K}$. The sample was heated by electron beam bombardment and temperatures were measured using an optical pyrometer (Ircon UX20P, emissivity 0.35 ). The clean Mo(110) surface was verified by low-energy electron diffraction (LEED) and STM before oxidation. Once a clean surface was obtained, the sample was oxidized at $1275 \mathrm{~K}$ in an oxygen atmosphere of $1 \times 10^{-7}$ mbar for 2 minutes. The quality of the resulting oxide structure was verified by LEED and STM. In order to obtain oxygen adatoms on the $\mathrm{MoO}_{2} / \mathrm{Mo}(110)$ surface the clean Mo(110) was oxidized at $1225 \mathrm{~K}$ in an oxygen atmosphere of $1 \times 10^{-7}$ mbar for 2 minutes and cooled down in an oxygen atmosphere.

Density functional theory (DFT) calculations were performed using the Vienna Ab-initio Simulation Package (VASP) program. VASP implements a projected augmented waves basis set ${ }^{27}$ and periodic boundary conditions. The electron exchange and correlation was simulated by local density approximation (LDA) pseudopotentials with a Ceperley-Alder exchange-correlation density functional. ${ }^{28}$

High temperature oxidation of the $\mathrm{Mo}(110)$ surface at $1275 \mathrm{~K}$ in an $\mathrm{O}_{2}$ atmosphere of $1 \times 10^{-7} \mathrm{mbar}$ for 2 minutes leads to the formation of an ultrathin $\mathrm{MoO}_{2}(010)$ layer. ${ }^{29,30}$ A typical STM image taken of the $\mathrm{MoO}_{2} / \mathrm{Mo}(110)$ surface is shown in Fig. 1a. The $\mathrm{MoO}_{2}(010)$ surface has an O-Mo-O trilayer structure and forms well-ordered oxide nanorows, separated by $2.5 \mathrm{~nm}$ (Fig. 1a). These rows follow the [1-1-3] direction of the Mo(110) substrate and appear in STM images as bright regions with dark depressions in between. Due to the formation of $\mathrm{MoO}_{2}$ nanorows, the surface represents an interesting nanostructured template suitable for the controlled adsorption of atoms, nanoclusters and molecules. ${ }^{22,31}$

In order to identify the correct model for the $\mathrm{MoO}_{2} / \mathrm{Mo}(110)$ surface that exhibits oxide nanorows similar to the STM image, DFT calculations have been performed. The Mo(110) surface was modeled by three layers of molybdenum. The lowest layer of molybdenum was constrained to simulate the bulk. An O-Mo-O trilayer, representing the ultrathin $\mathrm{MoO}_{2}$ film, was then placed on top of the three Mo layers. This slab was terminated by a vacuum region to simulate the surface. The resulting model of the 
$\mathrm{MoO}_{2} / \mathrm{Mo}(110)$ surface after relaxation is shown in Figs. 1c and d. To compare the DFT results with the STM images, the partial charge density of the relaxed system has been simulated in the range from the Fermi energy $\left(E_{F}\right)$ to 0.1 V. Figures $1 \mathrm{a}$ and $\mathrm{b}$ show an excellent agreement between the calculated and experimental STM images. This indicates that $\mathrm{MoO}_{2}(010)$ grows as a strained-commensurate layer on $\operatorname{Mo}(110)$ and is slightly corrugated (see Fig. 1c). Furthermore, the grooves between the oxide nanorows are due to the $\mathrm{MoO}_{2} / \mathrm{Mo}(110)$ surface topology.

Annealing the Mo(110) surface at a slightly lower temperature (1225 K) for 2 minutes and cooling down in an $\mathrm{O}_{2}$ atmosphere of $1 \times 10^{-7}$ mbar results in the formation of an oxygen-rich $\mathrm{MoO}_{2+\mathrm{x}} / \mathrm{Mo}(110)$ surface exhibiting oxygen adatoms. A typical STM image of the $\mathrm{MoO}_{2+\mathrm{x}} / \mathrm{Mo}(110)$ surface is shown in Fig. 2a, where oxygen adatoms appear as bright protrusions. Oxygen adatoms are adsorbed on top of the Mo oxide nanorows, which are visible in Fig. 2a, forming a perfectly aligned double row structure. The average distances between two adjacent oxygen adatoms along and perpendicular to the nanorow direction are approximately $1 \mathrm{~nm}$ and $1.2 \mathrm{~nm}$, respectively, and are dictated by the $\mathrm{MoO}_{2}(010)$ layer.

In order to simulate the $\mathrm{MoO}_{2+\mathrm{x}} / \mathrm{Mo}(110)$ system, two oxygen adatoms (blue spheres in Figs. 2c and d) were placed on the $\mathrm{MoO}_{2} / \mathrm{Mo}(110)$ surface. The positions of these adatoms on the surface were chosen on the basis of STM images. The resulting fully relaxed model of the $\mathrm{MoO}_{2+\mathrm{x}} / \mathrm{Mo}(110)$ surface is shown in Figs. 2c and d. It is noted that oxygen adatoms lead to the local formation of $\mathrm{MoO}_{3}$ clusters. As a result, the Mo atoms forming these clusters are pulled away from the surface (see Fig. 2c). The partial charge density calculated in the range from $\mathrm{E}_{\mathrm{F}}$ to $1.5 \mathrm{~V}$ (Fig. 2b) shows a very good agreement with experimental STM images.

Oxygen adatoms are stable on the $\mathrm{MoO}_{2} / \mathrm{Mo}(110)$ surface if imaged by STM at any negative bias or when a positive bias smaller than $1.5 \mathrm{~V}$ is applied to the sample. At positive biases greater than $1.5 \mathrm{~V}$, oxygen adatoms can be desorbed from the surface. Fig. 3 shows consecutive images taken from the same area of the $\mathrm{MoO}_{2+\mathrm{x}} / \mathrm{Mo}(110)$ surface after applying positive voltage pulses of $4 \mathrm{~V}$ (Figs. 3b, c and d) and $3 \mathrm{~V}$ (Fig. 3e) for a short time $(100 \mu \mathrm{s})$. A voltage pulse of $4 \mathrm{~V}$ results in the desorption of oxygen 
adatoms from a circular surface area $7 \mathrm{~nm}$ in diameter (approximately three oxide nanorows). In turn, the $3 \mathrm{~V}$ pulse removes oxygen atoms from a smaller area, with a diameter of $5 \mathrm{~nm}$.

The desorption of atoms from a surface by STM can occur by several mechanisms. The STM system consists of an atomically-sharp metal tip, a sub-nanometer sized vacuum gap, and a conducting sample upon which a bias voltage is placed. The bias voltage allows electrons to quantum-mechanically tunnel through the vacuum and these electrons can cause atoms to desorb. If these electrons are conducted away through the sample or tip (depending on the bias) without depositing their energy, this is termed elastic tunneling, however if the electrons lose energy through interactions with adsorbates or the surface, inelastic tunneling occurs. ${ }^{5,32-36}$ Inelastically tunneling electrons can cause the controlled excitation of adsorbed atoms or molecules, and can be used to manipulate or charge adsorbates or to break the bonds between molecular fragments. In this process low energy tunneling electrons (or holes) are injected to the atom (or molecule) located on a surface by positioning the tip above the target. The tunneling electrons' energy is transferred to an atom/molecule through a resonance state leading to various (single and multiple) excitations. ${ }^{33,37}$ In this process, the maximum energy possessed by the tunneling electrons can be controlled by adjusting the applied bias, and the probability of capture and the excitation rate can be varied by changing the tunneling current (i.e. the number of tunneling electrons per second).

Another consequence of having the voltage applied across such a small distance is that a large electric field is created between the sample and the tip. Such a field can be used to manipulate polarized atoms and molecules, which will experience either an attractive or repulsive force, depending on the bias polarity. ${ }^{38}$ In this paper we distinguish between these two phenomena by examining the effects of the bias voltage and the tip-sample separation on the desorption of adatoms.

The dependence of the threshold desorption voltage on the pulse length (Fig. 4) as well as the density of states associated with the oxygen adatom on the oxidized molybdenum surface (Fig. 5) favor 
the inelastic tunneling-induced desorption scenario. The dependence of the threshold voltage on the pulse duration measured at different tip-sample distances (Fig. 4) reveals two distinct features.

Firstly, the threshold voltage at all distances and pulse lengths is never lower than $1.5 \mathrm{~V}$. Similarly, the PDOS of the oxygen adatom exhibits unoccupied states just above $1.5 \mathrm{eV}$ (Fig. 5) and no unoccupied states below $1.5 \mathrm{eV}$. Intuitively, these two facts support one another; tunneling into these empty states can produce vibrational excitations which lead to the breaking of the chemical bonds between the oxygen adatom and $\mathrm{MoO}_{2}$ tri-layer structure underneath. The PDOS of the other $\mathrm{O}$ atoms in the model is shown for comparison, and it is noted that it does not exhibit the same peak at $1.5 \mathrm{~V}$, and instead shows a broad band of unoccupied states. Secondly, Fig. 4 demonstrates the strong dependence of the threshold voltage on the tip-sample distance. This is further evidence for the inelastic tunnelinginduced oxygen desorption theory, because higher voltages are required to reach the same tunneling current at larger tip-sample distances. The tunneling current should be above a critical value due to the finite vibrational lifetime for the oxygen adatom on $\mathrm{MoO}_{2} / \mathrm{Mo}(110)$ surface, as has been observed for the $\mathrm{Si}-\mathrm{H}$ system discussed in the first publications on desorption by inelastic electron tunneling. ${ }^{32}$

Because of the finite lifetime of the (previously) unoccupied oxygen adatom electron states above $1.5 \mathrm{eV}$, a minimum number of available tunneling electrons which can occupy these states is crucial to increase the probability of adatom desorption. The requirement of reaching a minimal current value rather than the cumulative number of electrons transferred leads to the different saturation threshold voltages observed at different tip-sample distances in Fig. 4. These different voltages likely correspond to the same tunneling current flowing through the oxygen adatom(s) at different Z-offsets. This critical tunneling current value is also responsible for the dependence of the area desorbed on the bias voltage at the same tip-sample distance (Fig. 3). Because of the strong exponential dependence of the current on the tip-sample distance, the tunneling current is maximal under the tip, while the number of electrons tunneling to neighboring surface atoms is substantially smaller. 
The length of time that the electron states above $1.5 \mathrm{eV}$ remain occupied by tunneling electrons can be insufficient for the initiation of vibrational excitations on distant oxygen adatoms when the voltage is below the threshold for that particular tunneling current. By increasing the bias voltage (and consequently, tunneling current), oxygen adatoms located further from the tip apex can become excited, leading to their desorption from the oxidized Mo(110) surface. Therefore the size of the area cleared of oxygen adatoms increases with increasing bias voltage when the tip-sample distance is held constant, as Figure 3 illustrates.

Controlled lithography via the desorption of atoms from surfaces due to inelastic electron tunneling has been previously demonstrated on the H-passivated $\mathrm{Si}(100)$ surface. ${ }^{32}$ Model calculations have indicated that the extremely high current densities present during STM can produce multiplevibrational excitations through inelastic electron tunneling, and in some cases the deposited energy can be sufficiently high to cause desorption. It was observed that, when the energy of incident electrons was greater than the threshold energy (achieved by applying a $6.5 \mathrm{~V}$ bias to the surface), the $\mathrm{H}$ atoms have been desorbed from the $\mathrm{Si}(100)$ surface at a nearly constant yield. ${ }^{32}$ Our data support the proposed mechanism of multiple-vibrational excitation as a result of inelastic electron tunneling. The observed threshold bias of $1.5 \mathrm{~V}$ for oxygen desorption indicates that the $\mathrm{O}-\mathrm{MoO}_{2}$ bond is weaker than the $\mathrm{H}-\mathrm{Si}$ bond.

In order to study the influence of an electric field on the extra-oxygen-molybdenum bond, DFT calculations were performed on a single $\mathrm{MoO}_{3}$ cluster to minimize the calculation time. The total energy of the cluster was recorded while simultaneously varying the electric field applied and the Mo-O bond length. The changes in the total energy of the system as a function of the extra-O-Mo distance calculated for electric fields of different strengths (up to $+/-1.25 \mathrm{eV} / \AA$ ) are shown in Fig. 6. Two different directions of electric field were applied parallel to the $\mathrm{O}-\mathrm{MoO}_{2}$ bond, corresponding to a positive and negative sample bias, respectively. From Figure 6 it is clear that the field alone is not sufficient to break the $\mathrm{O}-\mathrm{MoO}_{2}$ bond. Even at the very high field of $-1.25 \mathrm{eV} / \AA$ (which corresponds to a sample bias of 
approximately $-10 \mathrm{~V}$ ), the energy barrier which must be overcome to remove an adatom is reduced by only $40 \%$. Furthermore, the results for positive sample biases, when the desorption of oxygen adatoms has been observed experimentally, show no significant reduction of the energy barrier. This indicates that the electric field cannot be responsible for the desorption of oxygen adatoms. Note also that applying high bias voltages at distances far from the sample where the tunneling current was below the noise level in the STM did not initiate oxygen adatom desorption. This also highlights the importance of tunneling electrons rather than the electric field for the desorption of oxygen adatoms from the $\mathrm{MoO}_{2+\mathrm{x}} / \mathrm{Mo}(110)$ surface.

The use of tunneling electrons to desorb oxygen adatoms from the $\mathrm{MoO}_{2+\mathrm{x}} / \mathrm{Mo}(110)$ surface makes controlled STM lithography with nanometer resolution possible. Tip movement along the surface produces a nanoscale pattern, with a minimum feature size of just $3 \mathrm{~nm}$. By moving the STM tip in a controlled fashion, information can be written, read and erased by applying specific biases between the surface and the tip. Figure 7 shows an example of nanoscale writing, with patterns "written" at a sample bias voltage of $3 \mathrm{~V}$ and "read" at a bias voltage of $1.5 \mathrm{~V}$.

In summary, using scanning tunnelling microscopy and density functional theory calculations we have shown that the high-temperature oxidation of the Mo(110) surface under specific conditions results in an oxygen-rich $\mathrm{MoO}_{2+\mathrm{x}} / \mathrm{Mo}(110)$ surface, exhibiting perfectly-aligned double rows of oxygen adatoms. These adatoms can be removed from the surface by scanning (or pulsing) at positive sample biases greater than 1.5 V. Experimental data and DFT calculations suggest that inelastic electron tunneling is responsible for the adatom desorption. Tip movement along the surface can be used for controlled lithography at the nanoscale, with a minimum feature size of just $3 \mathrm{~nm}$. By moving the STM tip in a predetermined fashion, information can be written, read and erased by applying specific biases between the surface and the tip. 
ACKNOWLEDGMENT This work was supported by Science Foundation Ireland (Principal Investigator grant number 12/IA/1264, and Walton Visitor Award grant number 08/W.1/B2583). A.C. acknowledges support of the 7th European Community Framework Programme, STM topographic images were processed using WSxM software. ${ }^{39}$

\section{FIGURES}
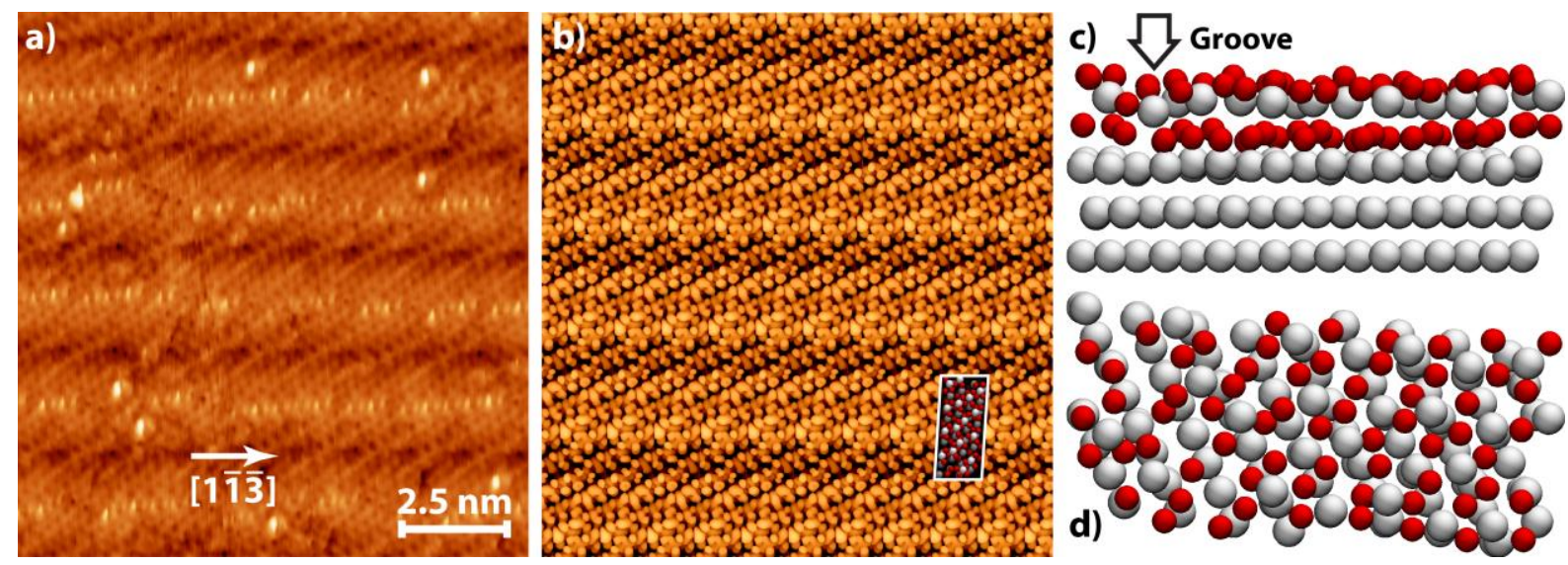

Figure 1. An experimental STM image $\left(13 \mathrm{~nm} \times 13 \mathrm{~nm}, \mathrm{~V}_{\mathrm{b}}=0.1 \mathrm{~V}, \mathrm{I}=0.10 \mathrm{nA}\right)$ (a) compared to the partial charge density of the relaxed $\mathrm{MoO}_{2} / \mathrm{Mo}(110)$ system, simulated in the range from $\mathrm{E}_{\mathrm{F}}$ to $0.1 \mathrm{~V}$ (b). The inset in (b) shows the surface unit cell. Side (c) and top (d) views of the calculated relaxed $\mathrm{MoO}_{2} / \mathrm{Mo}(110)$ structure. The $\mathrm{Mo}$ and $\mathrm{O}$ atoms are denoted by grey and red spheres, respectively. The arrow indicates the position of groove between the oxide nanorows. 

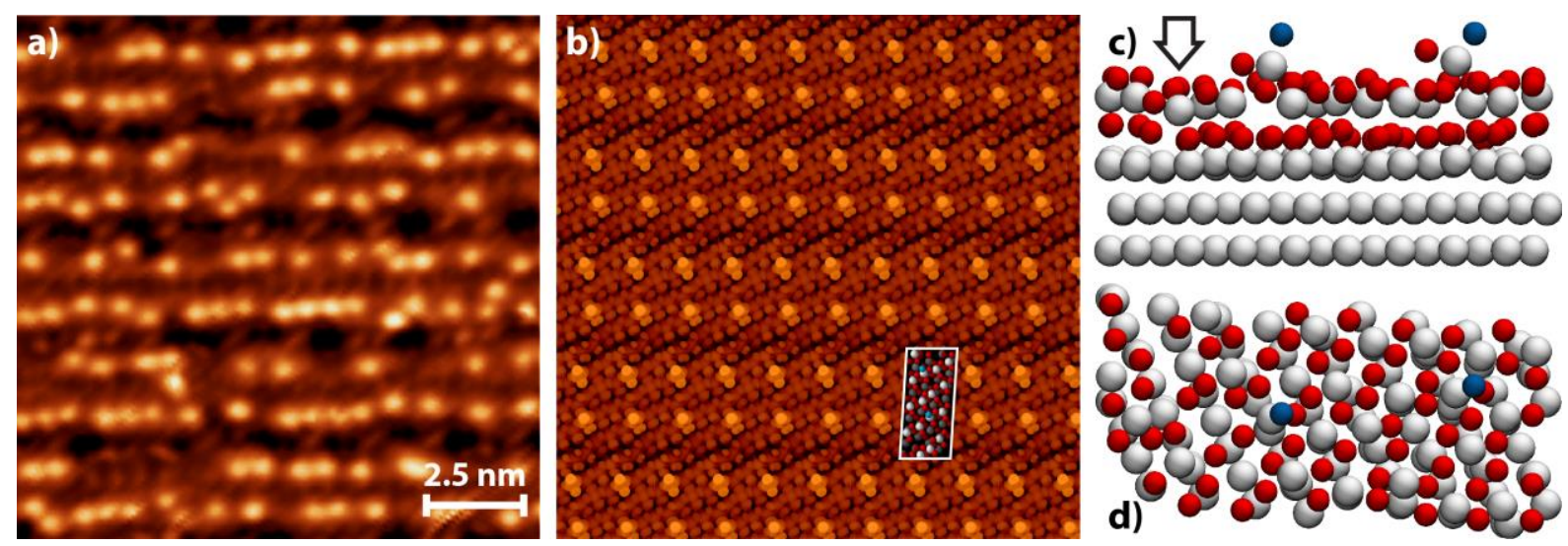

Figure 2. An experimental STM image $\left(12.5 \mathrm{~nm} \times 12.5 \mathrm{~nm}, \mathrm{~V}_{\mathrm{b}}=1.5 \mathrm{~V}, \mathrm{I}=1.0 \mathrm{nA}\right)$ (a) compared to the partial charge density of the relaxed $\mathrm{MoO}_{2+\mathrm{x}} / \mathrm{Mo}(110)$ system simulated in the range from $\mathrm{E}_{\mathrm{F}}$ to $1.5 \mathrm{~V}$ (b). The inset in (b) shows the surface unit cell. Side (c) and top (d) views of the calculated relaxed $\mathrm{MoO}_{2+\times} / \mathrm{Mo}(110)$ structure. The $\mathrm{Mo}$ and $\mathrm{O}$ atoms are denoted by grey and red spheres, respectively. The two oxygen adatoms are indicated by blue spheres. The arrow indicates the groove between oxide nanorows.
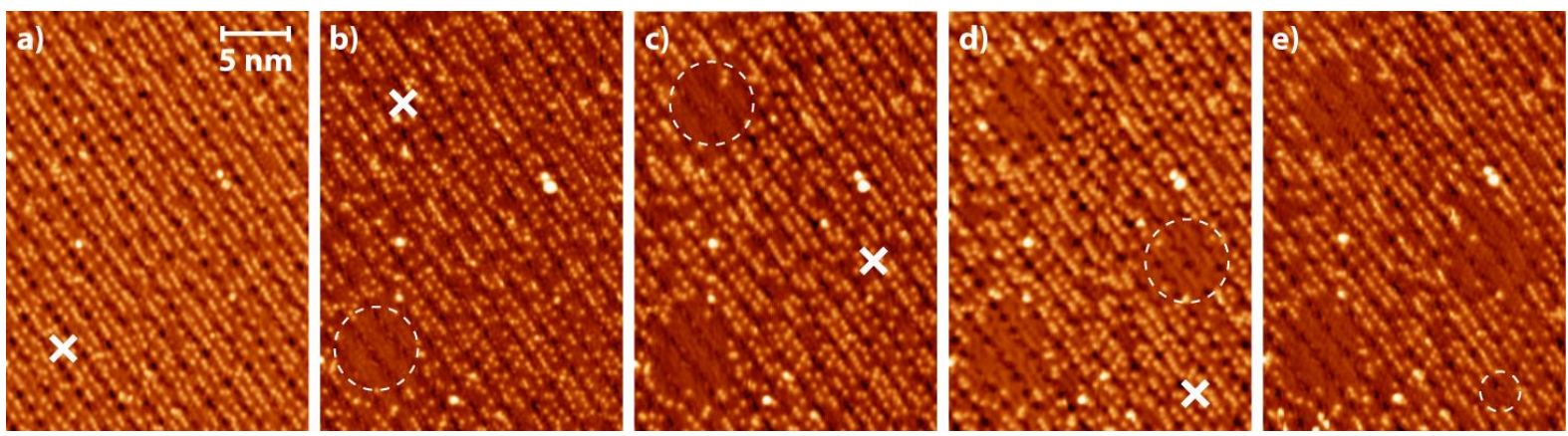

Figure 3. A sequence of low-temperature STM images taken from the same area of the $\mathrm{MoO}_{2+\mathrm{x}} / \mathrm{Mo}(110)$ surface $\left(\right.$ size $30 \mathrm{~nm} \times 40 \mathrm{~nm}, \mathrm{~V}_{\mathrm{b}}=1.5 \mathrm{~V}, \mathrm{I}=1.0 \mathrm{nA}$ ): (a) the initial STM image, (b)-(e) STM images after applying $4 \mathrm{~V}$ and $3 \mathrm{~V}$ pulses. White crosses on panels (a)-(d) show the pulse target positions, and the circles on panels (b)-(e) show areas cleared of adatoms. 


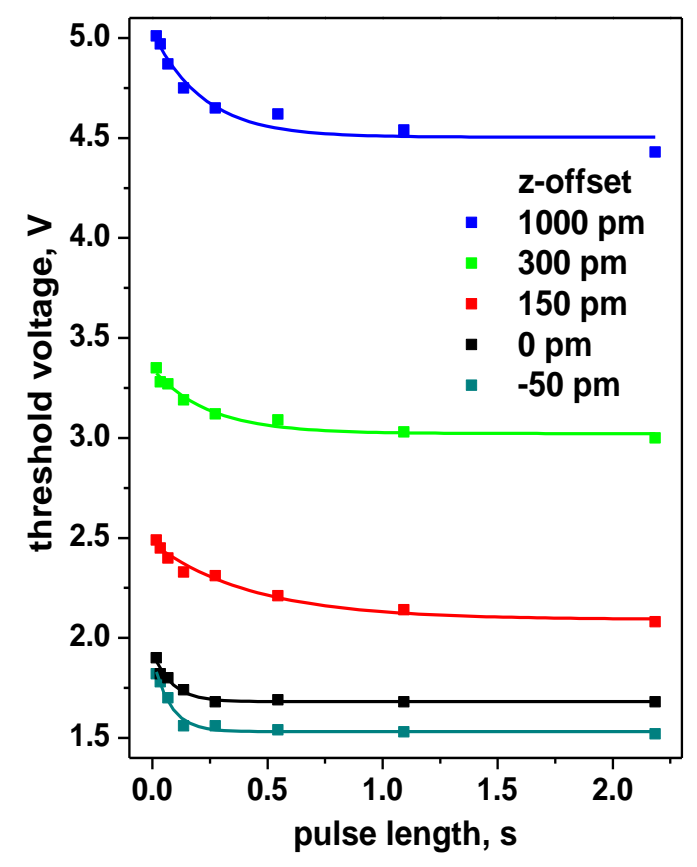

Figure 4. The threshold voltage is defined as the minimum voltage required to remove an oxygen adatom for a given pulse length. The threshold voltage depends strongly on the tip-sample separation, as well as the length of time the positive bias is applied. A z-offset of $0 \mathrm{pm}$ corresponds to the tip-to-surface distance required to achieve the tunneling conditions $\mathrm{V}_{\mathrm{b}}=1.5 \mathrm{~V}$ and $\mathrm{I}=1.0 \mathrm{nA}$, and a positive offset corresponds to a greater tip-surface distance.

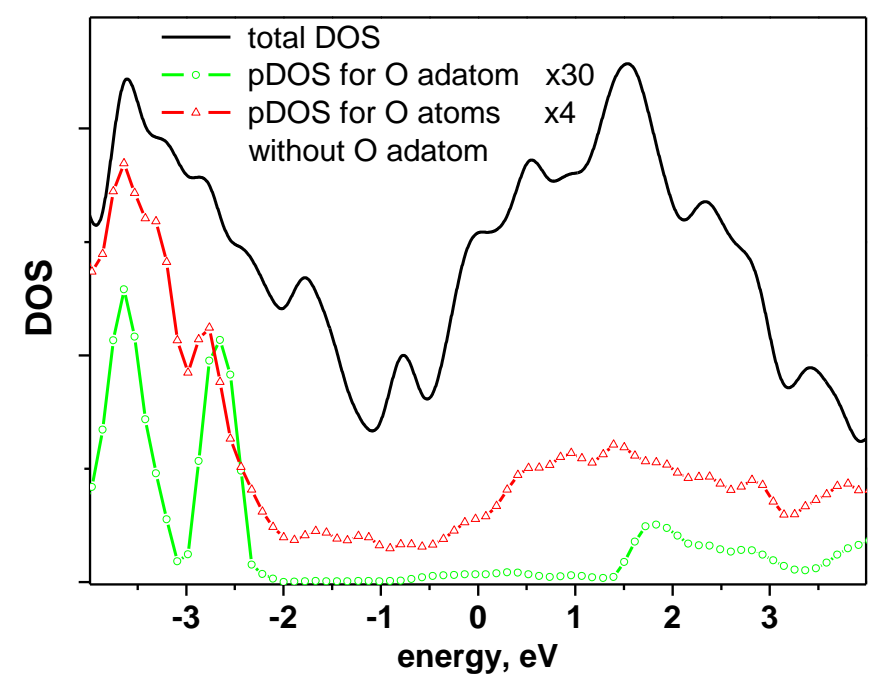

Figure 5. The total density of states (DOS) calculated for the full $\mathrm{MoO}_{2+\mathrm{x}} / \mathrm{Mo}(110)$ model shown in Figures $2 \mathrm{c}$ and $2 \mathrm{~d}$ is plotted in black. In green, the partial DOS for the oxygen adatom shows a peak in the unoccupied electron states above $1.5 \mathrm{eV}$. The DOS for all the $\mathrm{O}$ atoms in the system is shown in red 
for comparison, and in contrast, it contains a wide band of unoccupied states. All energies are set respective to the Fermi energy at $0 \mathrm{eV}$.

a)

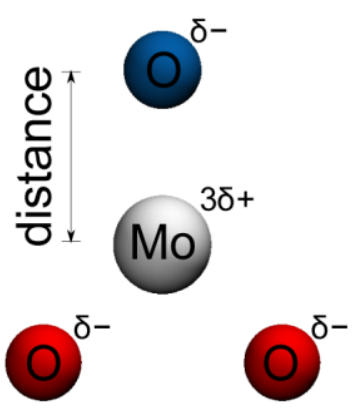

b) Positive sample bias

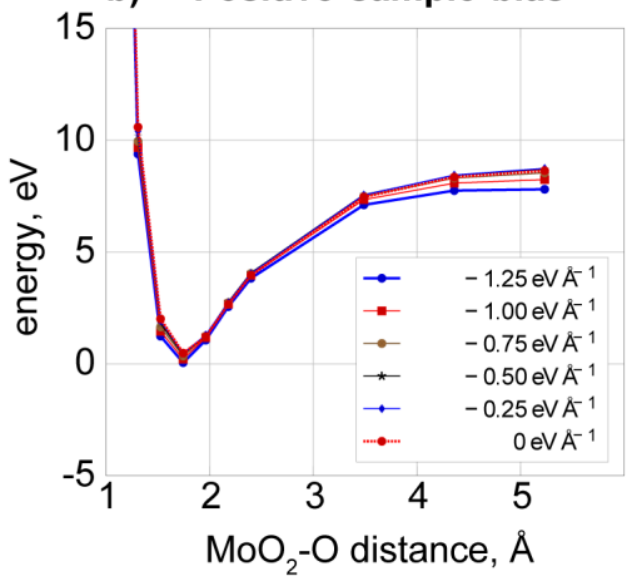

c) Negative sample bias

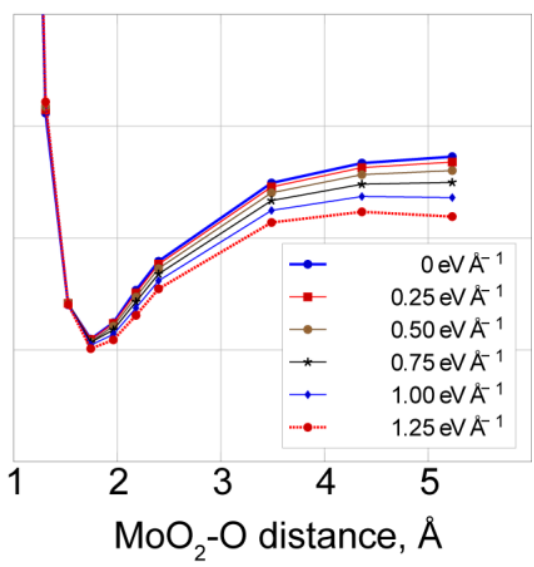

Figure 6. The total energy of the $\mathrm{MoO}_{3}$ cluster (a) as a function of the extra-O-Mo distance plotted for electric fields of different strength oriented along the extra-O-Mo bond. The results for positive and negative sample biases are shown in (b) and (c), respectively.
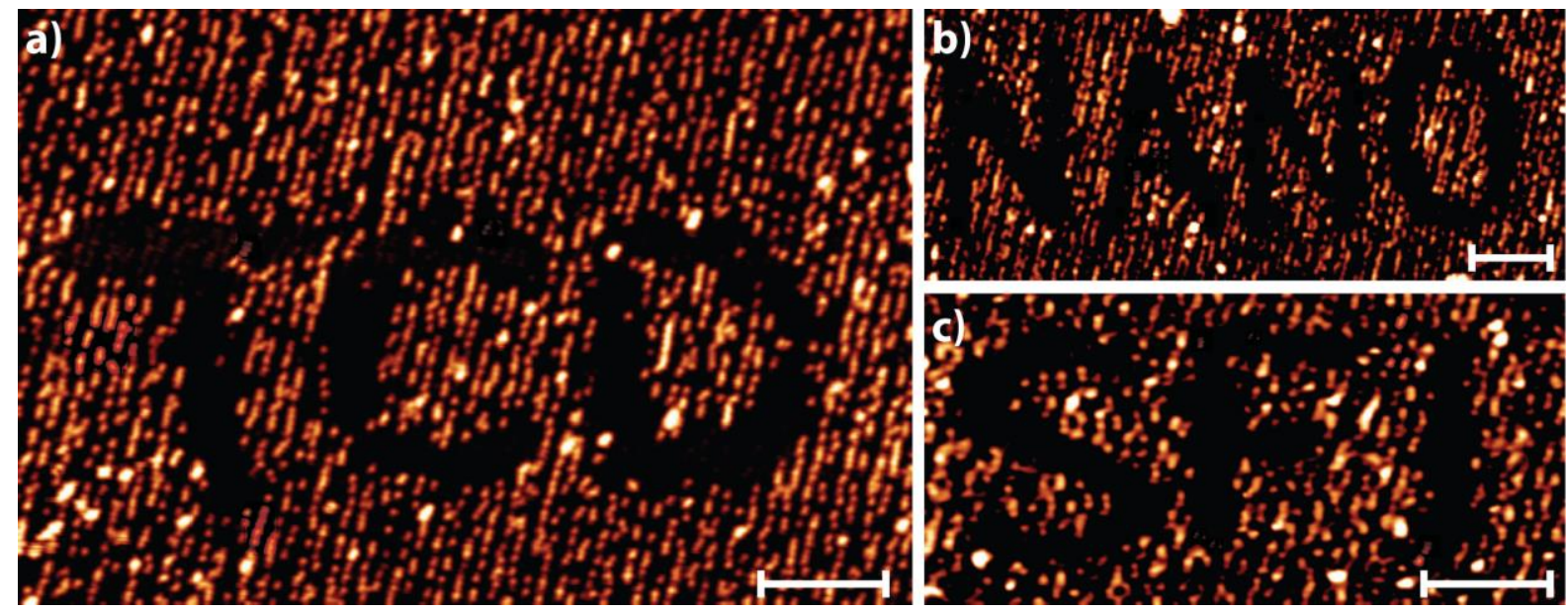

Figure 7. The words TCD, NANO and SFI written on the $\mathrm{MoO}_{2+\mathrm{x}} / \mathrm{Mo}(110)$ surface by moving the STM tip in a controlled fashion with a $3 \mathrm{~V}$ bias applied. The images were then recorded at a sample bias of $1.5 \mathrm{~V}$. All scale bars are $10 \mathrm{~nm}$ long. 


\section{REFERENCES}

(1) Binnig, G.; Rohrer, H.; Gerber, Ch.; Weibel, E. Phys. Rev. Lett. 1982, 49, 57-61.

(2) Ringger, M.; Hidber, H. R.; Schlögl, R.; Oelhafen, P.; Güntherodt, H.-J. Appl. Phys. Lett. 1985, $46,832-834$.

(3) Staufer, U.; Wiesendanger, R.; Eng, L.; Rosenthaler, L.; Hidber, H.R.; Güntherodt, H.-J.; Garcia, N. Appl. Phys. Lett. 1987, 51, 244-246.

(4) Eigler, D. M.; Schweizer, E. K. Nature 1990, 344, 524-526.

(5) Stroscio, J. A.; Eigler, D. M. Science 1991, 254, 1319-1326.

(6) Zeppenfeld, P.; Lutz, C. P.; Eigler, D. M. Ultramicroscopy 1992, 42-44, 128-133.

(7) Walsh M. A.; Hersam, M. C. Annu. Rev. Phys. Chem. 2009, 60, 193-216.

(8) Crommie, M. F.; Lutz, C. P.; Eigler, D. M. Science 1993, 262, 218-220.

(9) Pires, D.; Hedrick, J. L.; De Silva, A.; Frommer, J.; Gotsmann, B.; Wolf, H.; Despont, M.; Dürig, U.; Knoll, A. W. Science 2010, 328, 732-735.

(10) Wei, Z.; Wang, D.; Kim, S.; Kim, S.-Y.; Hu, Y.; Yakes, M. K.; Laracuente, A. R.; Dai, Z.; Marder, S. R.; Berger, C.; King, W. P.; de Heer, W. A.; Sheehan, P. E.; Riedo, E. Science 2010, $328,1373-1376$.

(11) Sugimura, H.; Kitamura, N.; Masuhara, H. Jpn. J. Appl. Phys. 1994, 33, L143-L145.

(12) Mühl, T.; Brückl, H.; Weise, G.; Reiss, G. J. Appl. Phys. 1997, 82, 5255-5258.

(13) Kolb, D. M.; Ullmann, R.; Will, T. Science 1997, 275, 1097-1099. 
(14) Piner, R. D.; Zhu, J.; Xu, F.; Hong, S.; Mirkin, C. A. Science 1999, 283, 661-663.

(15) Sugimura, H.; Nakagiri, N. Jpn. J. Appl. Phys. 1995, 34, 3406-3411.

(16) Sakurai, M.; Thirstrup, C.; Aono, M. Phys. Rev. B 2000, 62, 16167-16174.

(17) Wei, Yi-Min; Zhou, Xiao-Shun; Wang, Jin-Gang; Tang, Jing; Mao, Bing-Wei; Kolb, D. M. Small 2008, 4, 1355-1358.

(18) Hallam, T.; Reusch, T. C. G.; Oberbeck, L.; Curson, N. J.; Simmons, M. Y. J. Appl. Phys. 2007, $101,034305$.

(19) Cen, C.; Thiel, S.; Mannhart, J.; Levy, J. Science 2009, 323, 1026-1030.

(20) Hartwich, J.; Dreeskornfeld, L.; Heisig, V.; Rahn, S.; Wehmeyer, O.; Kleineberg, U.; Heinzmann, U. Appl. Phys. A 1998, 66, S685-S688.

(21) Krasnikov, S.A.; Murphy, S.; Berdunov, N.; McCoy, A.P.; Radican, K.; Shvets, I.V. Nanotechnology 2010, 21, 335301.

(22) Krasnikov, S. A.; Bozhko, S. I.; Radican, K.; Lübben, O.; Murphy, B.; Vadapoo, S.-R.; Wu, H.C.; Abid, M.; Semenov, V. N.; Shvets, I. V. Nano Res. 2011, 4, 194-203.

(23) Laursen, S.; Linic, S. Phys. Rev. Lett. 2006, 97, 026101.

(24) Santra, A. K.; Goodman, D. W. J. Phys.: Condens. Matter 2002, 14, R31-R62.

(25) Chaika, A. N.; Nazin, S. S.; Semenov, V. N.; Bozhko, S. I.; Lübben, O.; Krasnikov, S. A.; Radican, K.; Shvets, I. V. Europhys. Lett. 2010, 92, 46003.

(26) Chaika, A. N.; Nazin, S. S.; Semenov, V. N.; Orlova, N. N.; Bozhko, S. I.; Lübben, O.; Krasnikov, S. A.; Radican, K.; Shvets, I. V. Appl. Surf. Sci. 2013, 267, 219-223.

(27) Kresse, G.; Furthmüller, J. Phys. Rev. B 1996, 54, 11169-11186. 
(28) Ceperley, D. M.; Alder, B. J. Phys. Rev. Lett. 1980, 45, 566-569.

(29) Radican, K.; Berdunov, N.; Manai, G.; Shvets, I. V. Phys. Rev. B 2007, 75, 155434.

(30) Radican, K.; Berdunov, N.; Shvets, I. V. Phys. Rev. B 2008, 77, 085417.

(31) Lübben, O.; Krasnikov, S. A.; Preobrajenski, A. B.; Murphy, B. E.; Bozhko, S. I.; Arrora, S. K.; Shvets, I. V. J. Appl. Phys. 2012, 11, 07B515.

(32) Shen, T.-C.; Wang, C.; Abeln, G. C.; Tucker, J. R.; Lyding, J. W.; Avouris, Ph.; Walkup, R. E. Science 1995, 268, 1590-1592.

(33) Stipe, B. C.; Rezaei, M. A.; Ho, W.; Gao, S.; Persson, M.; Lundqvist, B. I. Phys. Rev. Lett. 1997, $78,4410-4413$.

(34) Stroscio, J. A.; Celotta, R. J. Science 2004, 306, 242-247.

(35) Bozhko, S. I.; Krasnikov, S. A.; Lübben, O.; Murphy, B. E.; Radican, K.; Semenov, V. N.; Wu, Han-Chun; Levchenko, E. A.; Chaika, A. N.; Sergeeva, N. N.; Shvets, I. V. Nanoscale 2013, 5, $3380-3386$

(36) Repp, J.; Meyer, G.; Olsson, F. E.; Persson, M. Science 2004, 305, 493-495.

(37) Gadzuk, J. W. Surf. Sci. 1995, 342, 345-358.

(38) Eigler, D. M.; Lutz, C. P.; Rudge, W. E. Nature 1991, 352, 600-603.

(39) Horcas, I.; Fernandez, R.; Gomez-Rodriguez, J. M.; Colchero, J.; Gomez-Herrero, J.; Baro, A.M. Rev. Sci. Instrum. 2007, 78, 013705. 